\title{
Toxicidade ocular causada pela cloroquina: relato de caso
}

\author{
Ocular toxicity caused by chloroquine:case report
}

\author{
Eliane Terumi Inada ${ }^{1}$ \\ Karina Paula Watanabe ${ }^{2}$ \\ Sérgio Gustavo Tanaka ${ }^{3}$ \\ Luís Antônio Sakakisbara ${ }^{4}$
}

\begin{tabular}{|l|}
\hline RESUMO \\
\hline Relatar um caso de toxicidade ocular causada pela cloroquina. Aferiu-se \\
a acuidade visual de ambos os olhos em tabela de Snellen. Foram realizados \\
biomicroscopia do segmento anterior, refração, oftalmoscopia, angiofluo- \\
resceinografia e retinografia numa paciente de 53 anos, sexo feminino, \\
portadora de artrite reumatóide. Fez uso de cloroquina por 6 anos e havia \\
parado há um ano, quando veio ao nosso serviço. Apresentava acuidade \\
visual corrigida de 20/200 e 20/40. À biomicroscopia do segmento anterior \\
apresentava lente intra-ocular no olho direito e catarata nuclear 1+/4+ no \\
olho esquerdo, com opacidade corneana subepitelial inferior ao eixo visual \\
em ambos os olhos. À oftalmoscopia, apresentava mácula com aspecto de \\
tacho batido (atrofia do epitélio pigmentar da retina). A angiofluoresceino- \\
grafia mostrou maculopatia com aspecto de olho de boi. Relata-se um caso \\
típico de ceratopatia e maculopatia causados pela cloroquina.
\end{tabular}

Descritores: Cloroquina/efeitos adversos; Cloroquina/toxicidade; Degeneração macular/ induzido quimicamente; Doenças retinianas/induzido quimicamente; Toxicidade de drogas; Fundo de olho; Mácula lútea; Relato de caso

\section{INTRODUÇÃO}

A cloroquina tem sido usada no tratamento de muitas doenças como malária, lupus eritematoso sistêmico e discóide, artrite reumatóide, porfiria cutânea, urticária solar. Seu efeito colateral mais grave é a retinopatia, cuja frequiência varia de 0,001 a $40 \%$, dependendo dos métodos e dos critérios diagnósticos ${ }^{(1-2)}$. O objetivo deste estudo é relatar um caso de toxicidade ocular causada pela cloroquina.

Para a avaliação da paciente, aferiu-se a acuidade visual de ambos os olhos em tabela de Snellen. Foram realizadas biomicroscopia do segmento anterior, refração, oftalmoscopia, angiofluoresceinografia e retinografia.

\section{RELATO DE CASO}

Paciente de 53 anos, sexo feminino, portadora de artrite reumatóide, fez uso de cloroquina por 6 anos e havia parado há um ano, quando veio ao nosso serviço. Apresentava acuidade visual em olho direito de 20/200 e em olho esquerdo de 20/40 sem melhora com refração. À biomicroscopia do segmento anterior apresentava lente intra-ocular no olho direito e catarata nuclear 1+/4+ no olho esquerdo, com córnea verticilata em ambos os olhos. À oftalmoscopia apresentava mácula com aspecto de tacho batido bilateral (atrofia do epitélio pigmentar da retina) (Figura 1). A angiofluoresceinografia mostrou uma área central hiperfluorescente resultado de defeito em janela do epitélio pigmentar da retina em ambos os olhos (Figura 2). 


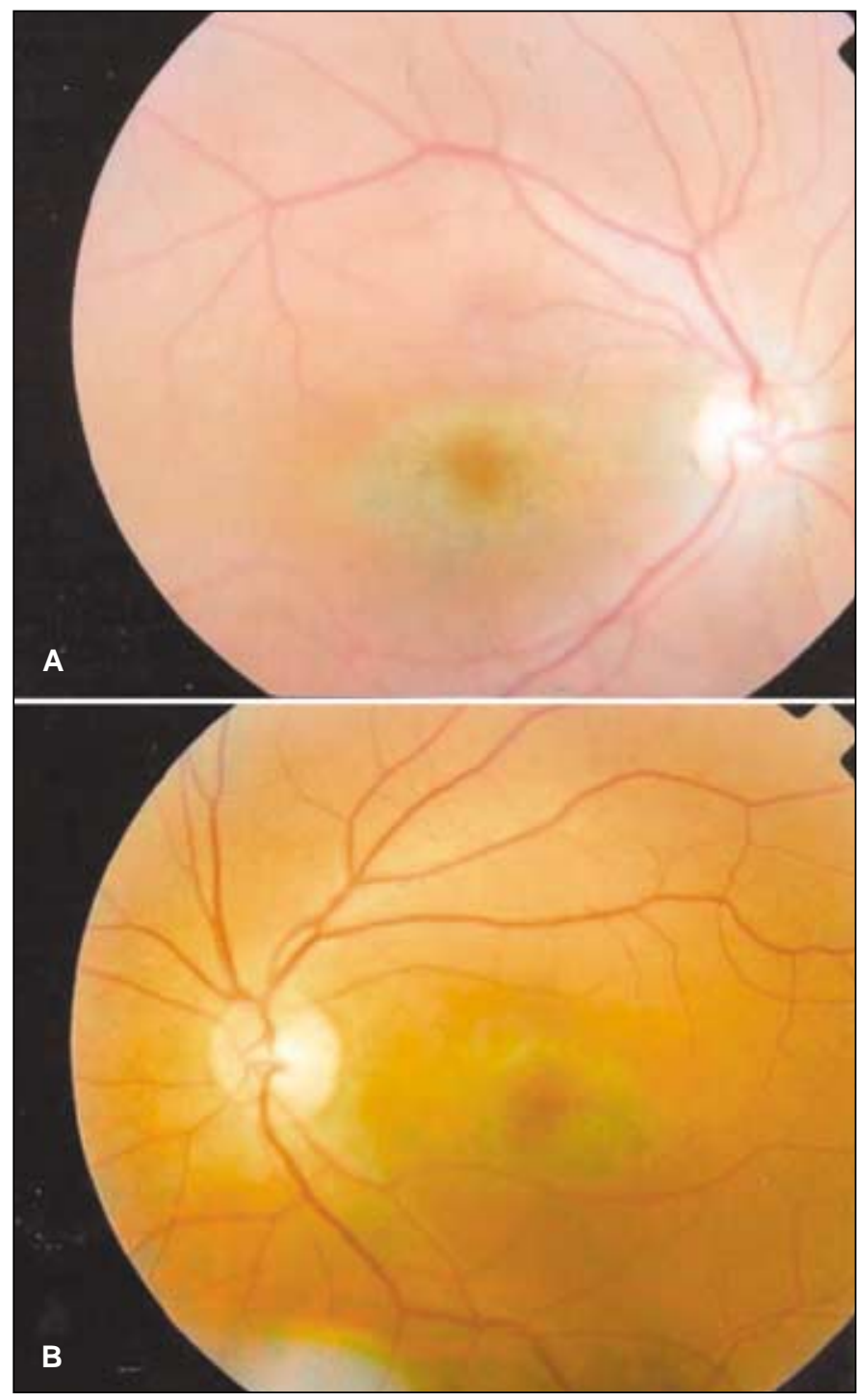

Figura 1 - Maculopatia em aspecto de olho de boi. A: olho direito; B: olho esquerdo

\section{COMENTÁRIOS}

Os dois efeitos colaterais mais importantes da cloroquina no olho são a retinotoxicidade e os depósitos corneanos. Embora incomuns, as alterações retinianas são potencialmente graves; e a ceratopatia, mais comum, é inócua. A droga é excretada lentamente e concentra-se nas estruturas oculares que contêm melanina, como o epitélio pigmentar da retina e coróide ${ }^{(3)}$.

A retinopatia pela cloroquina é irreversível e freqüentemente progressiva, estando relacionada com a dosagem cumulativa e à duração do tratamento ${ }^{(1,4-7)}$. Há relatos de detecção precoce e tardia variando de 9 meses após início do tratamento a 7 anos depois de cessado o mesmo ${ }^{(8-9)}$. Num estudo com pacientes usuários de cloroquina sem alterações no exame de fundo de olho, a sensibilidade ao contraste esteve dentro dos valores normais; porém a extensão das perdas na discriminação de cores foi positivamente correlacionada com a dose acumulada ${ }^{(10)}$.
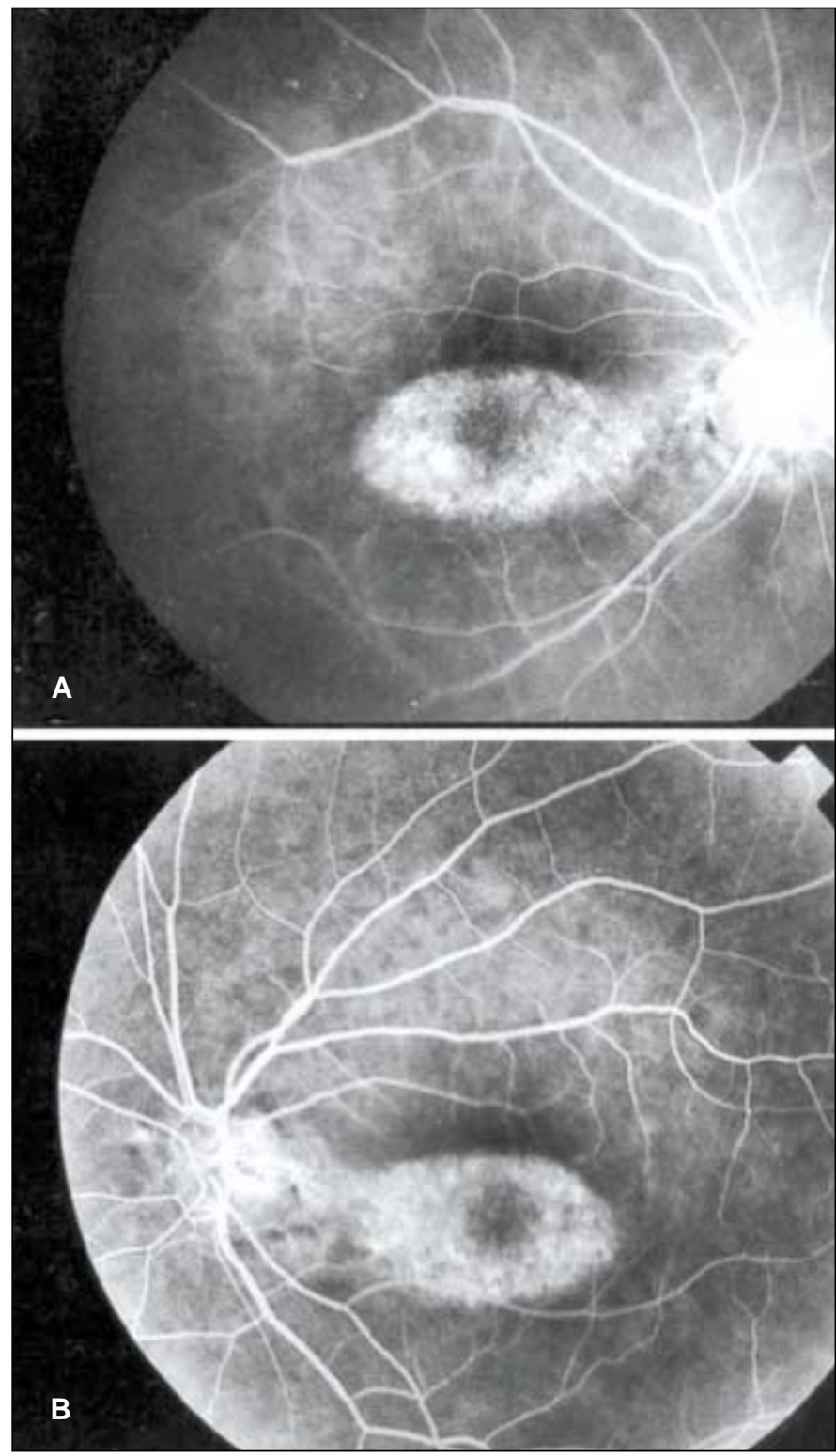

Figura 2 - Hiperfluorescência devido atrofia do epitélio pigmentar da retina na região macular. A: olho direito; B: olho esquerdo

A ceratopatia pode ser encontrada depois de 3 dias do início da terapia, sendo reversível com a descontinuidade da droga ${ }^{(8)}$. Não há prejuízo da acuidade visual e nenhuma diferença foi observada entre sexo, idade, duração do tratamento e dose acumulada ${ }^{(5,8,11)}$.

A dose diária da droga parece determinar o desenvolvimento da doença ocular, não devendo ultrapassar $4 \mathrm{mg} / \mathrm{kg} / \mathrm{dia}$. Para prevenir a superdosagem, o cálculo deve ser feito baseado no peso ideal do paciente e não no seu peso atual, além de ser necessária a monitorização de suas funções hepática e renal ${ }^{(12)}$.

A exposição solar aumenta o risco de retinopatia em pacientes tratados com cloroquina; portanto, óculos de sol devem ser recomendados ${ }^{(12)}$.

Recomenda-se exame oftalmológico de rotina antes do iní- 
cio do tratamento e a cada 6 meses, uma vez que a retinopatia pode ser detectada precocemente.

\section{ABSTRACT}

To report a case of ocular toxicity due to chloroquine. The best visual acuity was measured in both eyes with the Snellen chart. Slit-lamp examination of anterior segment, refraction, dilated fundus examination, fluorescein angiography and retinography was done in a 53-year-old patient, female, with rheumatoid arthritis. She had used chloroquine during 6 years and had stopped for 1 year when she came to our service. She had best corrected visual acuity of 20/200 and 20/40. Slit-lamp examination showed intraocular lens in right eye and nuclear cataract $(1+/ 4)$ in the other, and bilateral corneal subepithelial opacity inferior to the visual axis. Fundus examination showed macular area with retinal pigment epithelium atrophy. Fluorescein angiography showed a bull's eye maculopathy. Report of a typical keratopathy and maculopathy caused by chloroquine.

Keywords: Chloroquine/adverse effects; Chlroquine/toxicity; Macular degeneration/chemically induced; Retinal diseases/ chemically induced; Drug toxicity; Fundus oculi; Macula lutea; Case report

\section{REFERÊNCIAS}

1. Cox NH, Paterson WD. Ocular toxicity of antimalarial in dermatology: a survery of current practice. Br J Dermatol. 1994;131(6):878-82.

2. Finbloom DS, Silver K, Newsome DA, Gunkel R. Comparison of hydroxychloroquine and chloroquine use and the development of retinal toxicity. $\mathrm{J}$ Rheumatol. 1985;12(4):692-4.

3. Kanski JJ. Distúrbios maculares adquiridos. In: Kanski JJ. Oftalmologia clínica. 4 ed. Rio de Janeiro: Rio Méd Livros; 2004. p.437-9.

4. Martin LJ, Bergen RL, Dobrow HR. Delayed onset chloroquine retinopathy: case report. Ann Ophthalmol. 1978;10(6):723-6.

5. Fishman GA. Retinal toxicity with the use of chloroquine or hydroxychloroquine. In: Fishman GA. Principle and practice of clinical electrophysiology of vision. San Francisco; American Academy of Ophthalmology; 1991. p.594-9.

6. Nylander U. Ocular damage in chloroquine therapy. Acta Ophthalmol (Copenh). 1966;44(3):335-48.

7. Voipio H. Incidence of chloroquine retinopathy. Acta Ophthalmol (Copenh). 1966;44(3):349-54.

8. Puavilai S, Kunavisarut S, Vatanasuk M, Timpatanapong P, Sriwong ST, Janwitayanujit $\mathrm{S}$, et al. Ocular toxicity of chloroquine among Thai patients. Int J Dermatol. 1999;38(12):934-7.

9. Ehrenfeld M, Nesher R, Merin S. Delayed - onset chloroquine retinopathy. Br J Ophthalmol. 1986;70(4):281-3.

10. Ventura DF, Silveira LCL, Nishi M,Costa MF, Gualtieri M, Santos RMA, et al. Color vision loss in patients treated with chloroquine. Arq Bras Oftalmol. 2003;66(Supl 5):9-15.

11. Easterbrook M. Ocular effects and safety of antimalarial agents. Am J Med. 1988;85(4A):23-9.

12. Mackenzie AH. Dose refinements in long-therm therapy of rheumathoid arthritis with antimalarials. Am J Med. 1983;75(1A):40-5. 